\title{
Ototoxicity of cisplatin plus standard radiation therapy vs. accelerated radiation therapy in glioblastoma patients
}

\author{
Nicole E. Marshall ${ }^{1}$, Karla V. Ballman ${ }^{1}$, John C. Michalak ${ }^{2}$, Paula J. Schomberg ${ }^{1}$, Gary V. Burton ${ }^{3}$ \\ Howard M. Sandler ${ }^{3}$, Terrence L. Cascino ${ }^{1}$, Kurt A. Jaeckle ${ }^{4}$ and Jan C. Buckner ${ }^{1}$ \\ ${ }^{1}$ Mayo Clinic and Mayo Foundation, Rochester, MN, 55905, USA; ${ }^{2}$ Siouxland Hematology-Oncology Associates, \\ Sioux City, IA, 51105, USA; ${ }^{3}$ SWOG - University of Michigan, Ann Arbor, MI, 48108-0010, USA; ${ }^{4}$ Mayo Clinic \\ Jacksonville, Jacksonville, FL, 32224, USA
}

Key words: accelerated radiation therapy, glioblastoma, ototoxicity of cisplatin

\begin{abstract}
Summary
Purpose: To assess the effect of cisplatin (CDDP) plus concurrent radiation therapy on hearing loss. Methods: 451 patients with glioblastoma multiforme (GBM) were randomly assigned after surgery to: Arm A: Carmustine (BCNU) + standard radiation therapy (SRT); Arm B: BCNU + accelerated radiation therapy (ART: 160 cGy twice daily for 15 days); Arm C: CDDP + BCNU + SRT; or Arm D: CDDP + BCNU + ART. Patients on arms $\mathrm{C}$ and $\mathrm{D}$ received audiograms at baseline, and prior to the start of RT, and prior to cycles 3 and 6 . Otologic toxicities were recorded at each visit. Results: $56 \%$ of patients had hearing loss at baseline. $13 \%$ and $50 \%$ of patients experienced worsening ototoxicity after 1 year of treatment in arms A and B vs. C and D, respectively, with $13 \%$ of those on arms C and D experiencing significant ototoxicity ( $\geq$ grade 3 ) at 6 months. Increasing age was associated with an increased risk of ototoxicity. Conclusions: Increased exposure to CDDP increases the risk of ototoxicity over time. Older patients are more susceptible to hearing loss with CDDP. The low proportion of patients with clinically significant ototoxicity suggests that baseline screening is unnecessary in GBM patients.
\end{abstract}

\section{Introduction}

Cisplatin (CDDP) is a chemotherapeutic agent used in the treatment of a variety of malignancies and is a wellknown ototoxic agent. Histopathologic evidence suggests that CDDP first destroys outer hair cells in the organ of Corti on the basal turn of the cochlea [1,2]. The outer hair cells are responsible for sensing higher frequencies, and their loss explains the most common otologic toxicity caused by cisplatin - high frequency sensorineural hearing loss [3]. With increased cumulative doses, damage to inner hair cells can occur, resulting in hearing loss at lower frequencies, including conversational speech ranges [1,4], additional damages can include decreased spiral ganglion cells and atrophy of stria vascularis $[4,5]$.

Cranial radiation can contribute to conductive hearing loss by inducing changes to the middle and external ear, [6] primarily by increasing the incidence of serous effusions [5]. The sensorineural aspect of hearing has generally been thought to be fairly resistant to radiation damage, although once damage occurs, it is more likely permanent [6]. One study found that of patients with parotid tumors treated with irradiation, 50\% had clinically relevant hearing loss, and in $33 \%$, this loss was permanent and affected quality of life [7]. An increased incidence of hearing loss has been found when CDDP is given following cranial radiation therapy in children, suggesting a synergistic toxic effect $[8,9]$. This does not appear to hold true when CDDP is given prior to radiotherapy.

One treatment regimen for glioblastoma multiforme (GBM) consists of a combination of surgery, BCNU, and RT. CDDP has been found to have anti-tumor activity against recurrent glioma with intravenous administration [10]. It has also been observed to remain in tissues for extended periods of time following administration [11]. This may account for its action as a radiosensitizer of hypoxic cells and its ability to potentiate post-RT cell kill, [12] thus increasing the effectiveness of the radiation therapy. However, the combination of CDDP and cranial radiation has the potential to cause serious ototoxicity [13]. In a recent clinical trial, this combination was evaluated to determine its effectiveness in prolonging survival of patients with GBM compared to standard treatment [14]. As a component of that study, this trial was designed to assess the ototoxicity of this treatment regimen.

In this study, we set out to examine the frequency and severity of otologic complaints, including hearing loss, tinnitus, and serous otitis media, in patients diagnosed with GBM and undergoing treatment with either standard therapy or chemotherapy including CDDP. Based on the study design, it is also possible to infer the time period during which hearing loss most commonly occurred, either during treatment with CDDP alone, with radiation alone, or with a combination of the two treatment modalities. We used serial 
pure tone audiograms to determine the incidence and severity of any hearing impairment in patients treated with CDDP.

\section{Materials and methods}

\section{Protocol therapy}

Patients with newly diagnosed histologically confirmed glioblastoma multiforme were randomly assigned after surgical biopsy or excision to one of four treatment arms as shown in Figure 1. Treatment began within 4 weeks of surgery. Patients on arms A and B received $200 \mathrm{mg}$ / $\mathrm{m}^{2}$ intravenous BCNU once every 8 weeks for 6 cycles, and either SRT consisting of $180 \mathrm{cGy}$ once a day for 36 days or ART with 160 cGy twice daily for 15 days. Patients on arms $\mathrm{C}$ and D received $50 \mathrm{mg} / \mathrm{m}^{2}$ intravenous BCNU days $1-3$, every 8 weeks for 2 cycles, then $200 \mathrm{mg} / \mathrm{m}^{2}$ once every 8 weeks for 4 cycles. In addition, they received $30 \mathrm{mg} / \mathrm{m}^{2}$ intravenous CDDP days $1-3$ and 29-31 every 8 weeks for 2 cycles and either SRT or ART. Patients on arms C and D had 8 weeks of chemotherapy prior to initiating RT [14].

\section{Patient evaluation}

Serial audiograms were requested of all patients receiving CDDP starting with a baseline audiogram to be scheduled as soon as the treatment arm was assigned. Although testing was preferably prior to the initiation of treatment, commencement of treatment was not to be delayed. Additional audiograms were to be completed prior to the start of RT, prior to cycles 3 and $6 \mathrm{BCNU}$, and as clinically indicated thereafter.

\section{Assessment of toxicity}

At each evaluation, toxicities to be graded included (1) neuro-hearing and (2) acute radiation toxicity to the ear. These were evaluated per National Cancer Institute (NCI) Common Toxicity Criteria grading version 2.0, which defines 'neuro-hearing' toxicity as grade 1: asymptomatic, hearing loss on audiometry only; grade 2: tinnitus; grade 3: hearing loss interfering with function but correctable with hearing aid; grade 4: deafness not correctable; and 'acute radiation toxicity to the ear' as grade 1: mild external otitis with erythema, pruritis, secondary to dry desquamation not requiring medication with audiogram unchanged from baseline; grade 2: moderate external otitis requiring topical medication/serous otitis medius/hypoacusis on testing only; grade 3: severe external otitis with discharge or moist desquamation/symptomatic hypoacusis/tinnitus, not drug-related; and grade 4: deafness.

CDDP was to be discontinued if grade 3 neurohearing toxicity was recorded, and the patient was to continue on BCNU and RT. Quality control of toxicity monitoring was verified by adhering to NCI guidelines requiring $10 \%$ of all cases accrued to be audited. In addition, each case was individually reviewed to insure that all institutions correctly applied the NCI common toxicity grading scale.

\section{Statistical analysis}

Categorical variables were summarized by the observed frequency and percent. Comparisons of categorical variables between two groups were performed with a $\chi^{2}$ test. Continuous variables were summarized using mean $\pm \mathrm{SD}$ as well as median and range (minimum to maximum values). A rank-sum test was used for comparisons of continuous variables between groups. All time-to-event outcomes were measured from date of study enrollment to date of event or last follow-up. Time-to-event outcomes were estimated via the Kaplan-Meier method [15]. Comparisons of KaplanMeier estimated time-to-event experiences between two groups were done with the log-rank test [16]. Univariable Cox proportional hazards models [17] were used to assess the association between baseline patient characteristics (age, gender, performance score, extent of resection, baseline anti-convulsant treatment, baseline corticosteroid treatment, family history of brain tumors), baseline pure tone hearing measures (250, 500, 1000, 2000, 3000, 4000, 6000, and $8000 \mathrm{~dB}$ ), and treatment arm (C vs. D). No multivariable Cox modeling was performed due to the small number of events. All tests were two-sided and a $P$-value $\leq 0.05$ was considered statistically significant.

Arm A: Standard radiation

therapy + carmustine

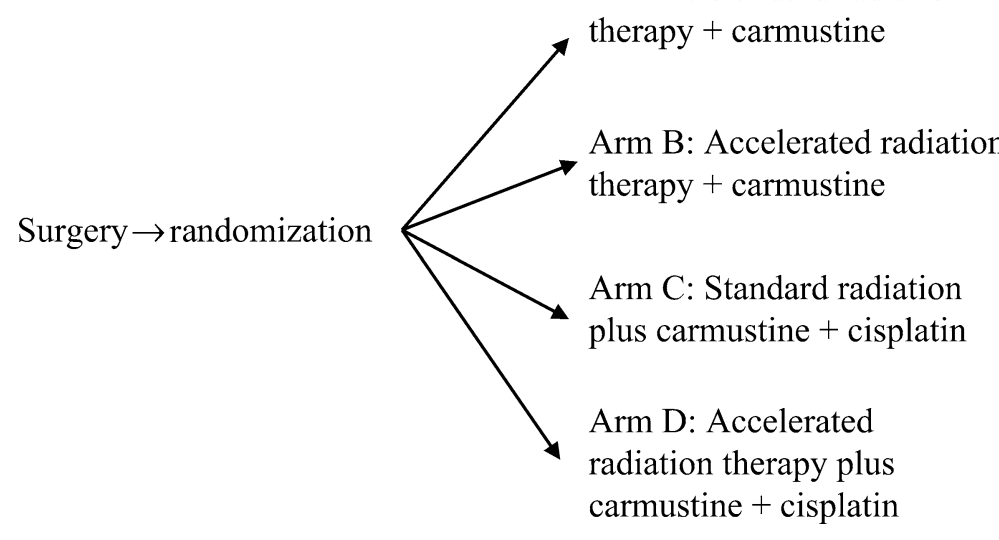

Figure 1. Treatment schedule. 


\section{Results}

A total of 230 patients were enrolled on arms C and D. Of these, $176(77 \%)$ received a baseline audiogram -86 (74\%) on arm C and $90(80 \%)$ on arm D (see Table 1); these patients comprised the study group. Patients who received baseline audiograms were slightly older than those who did not $(56 \pm 12$ years vs. $53 \pm 12$ years, respectively), although this difference did not achieve statistical significance $(P$-value $=0.09)$. In addition, patients who received baseline audiograms tended to have a worse performance score than patients who did not have a baseline audiogram, although this difference also did not quite achieve statistical significance $(P$-value $=0.051)$. There were no other obvious differences between patients who received a baseline audiogram and those who did not. The study group consisted of $66(37 \%)$ females and $110(63 \%)$ males. Most of these patients had a subtotal $(57 \%)$ or gross total resection $(24 \%)$. The majority were being treated with corticosteroids and/or anti-convulsants at baseline.

Table 1. Baseline patient characteristics

\begin{tabular}{|c|c|c|c|}
\hline Characteristics & $\begin{array}{l}\text { Individuals } \\
\text { with baseline } \\
\text { audiograms } \\
N=176\end{array}$ & $\begin{array}{l}\text { Individuals with } \\
\text { no baseline } \\
\text { audiogram } \\
N=54\end{array}$ & $P$-value \\
\hline Arm, $n(\%)$ & & & 0.28 \\
\hline $\mathrm{C}$ & $86(74)$ & $31(26)$ & \\
\hline $\mathrm{D}$ & $90(80)$ & $23(20)$ & \\
\hline Gender, $n(\%)$ & & & 1.00 \\
\hline Female & $66(37)$ & $20(37)$ & \\
\hline Male & $110(63)$ & $34(63)$ & \\
\hline Age, years & & & 0.090 \\
\hline Mean $\pm \mathrm{SD}$ & $55.8 \pm 11.5$ & $52.8 \pm 11.8$ & \\
\hline Median (min, $\max )$ & $56.0(22,79)$ & $52.8(29,78)$ & \\
\hline Age groups, $n(\%)$ & & & 0.075 \\
\hline$\leq 40$ years & $16(9)$ & $10(19)$ & \\
\hline $40+$ to 60 years & $96(55)$ & $31(57)$ & \\
\hline$>60$ years & $64(36)$ & $13(24)$ & \\
\hline PS, $n(\%)$ & & & 0.051 \\
\hline 0 & $59(34)$ & $28(52)$ & \\
\hline 1 & $93(53)$ & $20(37)$ & \\
\hline 2,3 or 4 & $24(14)$ & $6(11)$ & \\
\hline $\begin{array}{l}\text { Extent of } \\
\text { resection, } n(\%)\end{array}$ & & & 0.11 \\
\hline Biopsy & 33 (19) & $13(24)$ & \\
\hline STR & $100(57)$ & $22(41)$ & \\
\hline GTR & $43(24)$ & $19(35)$ & \\
\hline $\begin{array}{l}\text { Baseline } \\
\text { steroids, } n(\%)\end{array}$ & & & 0.45 \\
\hline Yes & $141(81)$ & $41(76)$ & \\
\hline No & 34 (19) & $13(24)$ & \\
\hline Missing & 1 & 0 & \\
\hline $\begin{array}{l}\text { Baseline anti- } \\
\text { convulsants, } n(\%)\end{array}$ & & & 0.47 \\
\hline Yes & $126(73)$ & $41(79)$ & \\
\hline No & $47(27)$ & $11(21)$ & \\
\hline Missing & 3 & 2 & \\
\hline $\begin{array}{l}\text { Family } \mathrm{hx} \text { of brain } \\
\text { tumor, } n(\%)\end{array}$ & & & 0.25 \\
\hline Yes & $11(7)$ & $6(11)$ & \\
\hline No & $158(93)$ & $48(89)$ & \\
\hline Missing & 7 & & \\
\hline
\end{tabular}

Table 2 summarizes the audiograms that were obtained. Of the 176 patients, 161 went on to receive RT, 78 were on arm $\mathrm{C}$ and received SRT along with CDDP and BCNU and the remaining 83 were assigned to arm D and received ART along with CDDP and BCNU. Audiograms prior to RT were obtained from 85 $(53 \%)$ of the 161 patients with baseline audiograms who received RT: $48(62 \%)$ on arm C and $37(44 \%)$ on arm D. Although audiograms were stipulated by the protocol prior to cycle 3 (immediately post-RT) and prior to cycle 6 , only 19 audiograms were obtained post-radiotherapy from the 142 patients with baseline audiograms who started cycle 3: $3(4 \%)$ from arm C and $16(22 \%)$ from arm D.

On baseline audiograms, $99(56 \%)$ of patients had some degree of hearing loss, defined as any abnormality on an audiogram. Of these 99 patients, 84 had loss in both ears, 7 had loss only in the left ear, and 8 had loss only in the right ear. The majority of patients with hearing loss $(70 \%)$ had losses in frequencies above 3000 dB. After 8 weeks of treatment with CDDP and BCNU and prior to the initiation of RT treatment, 54 (61\%) of the patients who had an audiogram prior to radiotherapy had some degree of hearing loss. Of the19 patients who had an audiogram post-radiotherapy, 12 (63\%) had some degree of hearing loss. There was no evidence of a difference in hearing loss between arms C and D.

Auditory/hearing adverse events, defined as hearing loss, otitis, tinnitus, and RT-ear, of grade 1 or higher were more frequent in patients on arms $\mathrm{C}$ or $\mathrm{D}$ compared to patients on arms $\mathrm{A}$ or $\mathrm{B}$. There was no evidence of a difference in hearing adverse events between arms $\mathrm{C}$ and D. Forty-one of the $117(36 \%)$ patients on arms $C$ and 43 of the 113 patients on arm D (38\%) reported at least one hearing related adverse event of grade 1 or higher. By comparison, 13 of the $111(12 \%)$ of patients on arm A and 13 of the $110(12 \%)$ on arm B reported at least one hearing related adverse event of grade 1 or higher. At 6 months, 15\% (95\% CI: 9-21) of patients on arms A or B experienced a hearing related adverse event of grade 1 or higher compared to $45 \%$ (95\% CI: $36-52)$ of patients on arms C or D (Figure 2). This difference was statistically significant $(P$-value $<0.001)$. The most commonly reported hearing related adverse events on arms C and D were hearing loss followed by tinnitus. Defining serious hearing related adverse events as grade 3 or higher, there was no apparent difference in the occurrence of serious hearing related adverse events between arms $\mathrm{A} / \mathrm{B}$ and $\operatorname{arms} \mathrm{C} / \mathrm{D}(P$-value $=0.19)$.

Table 2. Summary of audiograms

\begin{tabular}{lll}
\hline Characteristics & Arm C & Arm D \\
\hline Number enrolled & 117 & 113 \\
$\quad$ No. (\%) with baseline audiograms & $86(74)$ & $90(80)$ \\
Number with baseline audiogram & 78 & 83 \\
who received RT & & \\
$\quad$ No. (\%) with audiogram prior to RT & $50(64)$ & $38(46)$ \\
Number who started cycle 3 with & 67 & 73 \\
baseline audiogram & & $16(22)$ \\
$\quad$ No. (\%) with audiograms post-RT & $3(4)$ & \\
\hline
\end{tabular}




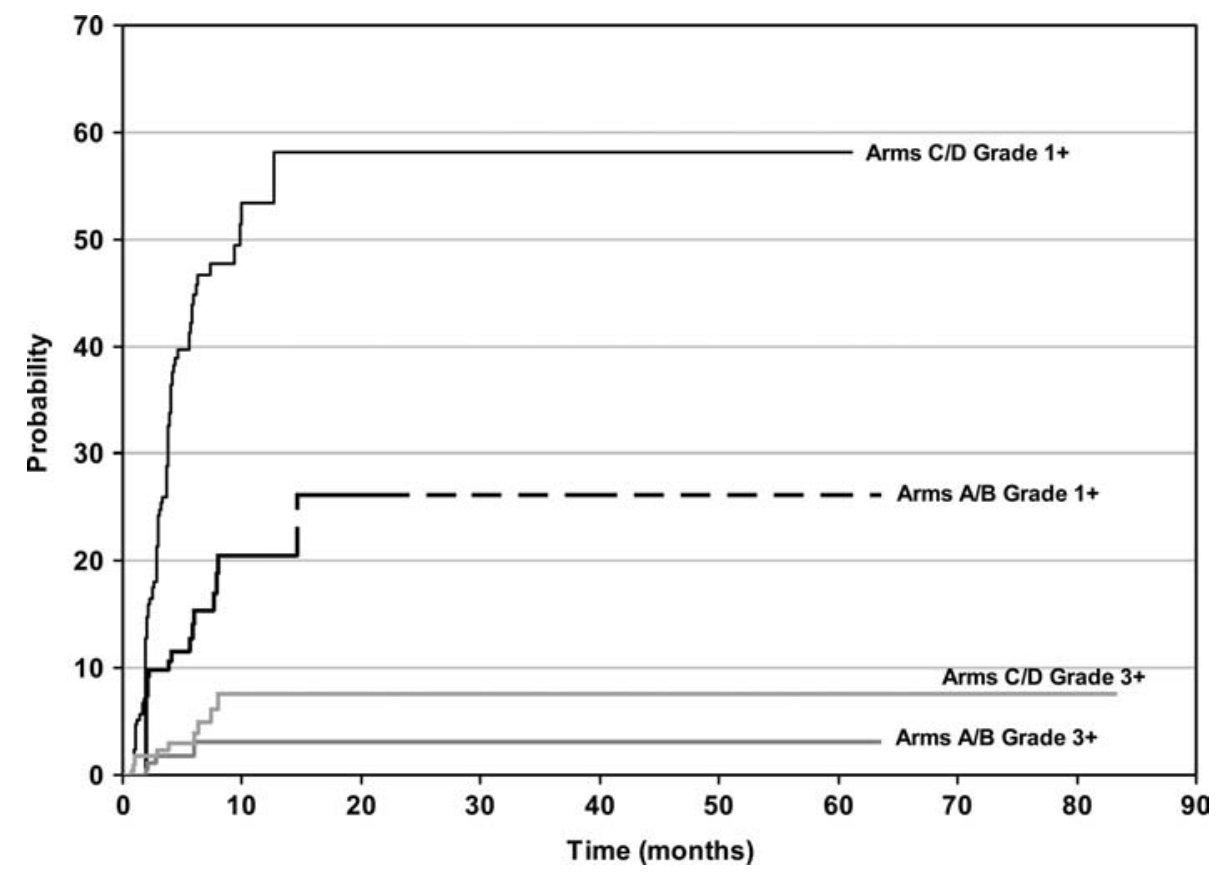

Figure 2. Hearing-related adverse events by treatment arm and severity.

Specifically, 3\% (95\% CI: 0-6) of the patients on arms A or B experienced a serious adverse event by 6 months compared to $4 \%(95 \% \mathrm{CI}: 1-7)$ of patients on arms $\mathrm{C}$ or D (Figure 2).

Conductive hearing loss was much less frequent than sensorineural hearing loss at baseline. Only $3(2 \%)$ patients had documented conductive hearing loss at baseline compared to $51(29 \%)$ patients with sensorineural loss. These percentages increased after CDDP + BCNU therapy to 6 of $88(7 \%)$ with conductive hearing loss and 32 of $88(36 \%)$ with sensorineural hearing loss. Although the number of patients with a post-RT audiogram was small, it does not appear as though these percentages increased post-RT: 0 of $19(0 \%)$ conductive hearing loss and 6 of $19(32 \%)$ sensorineural.

Figure 3 summarizes the rate of different definitions of hearing loss for patients on arms $\mathrm{C}$ and $\mathrm{D}$. The three measures are (a) a loss documented by an audiogram in any pure tone range, (b) a clinically significant hearing loss - a documented loss on audiogram of greater than $20 \mathrm{~dB}$ at any frequency less than $3000 \mathrm{~dB}$, and (c) a clinically significant hearing loss and/or a hearing related adverse event of grade 3 or higher. These measures are not meant to be compared to each other,

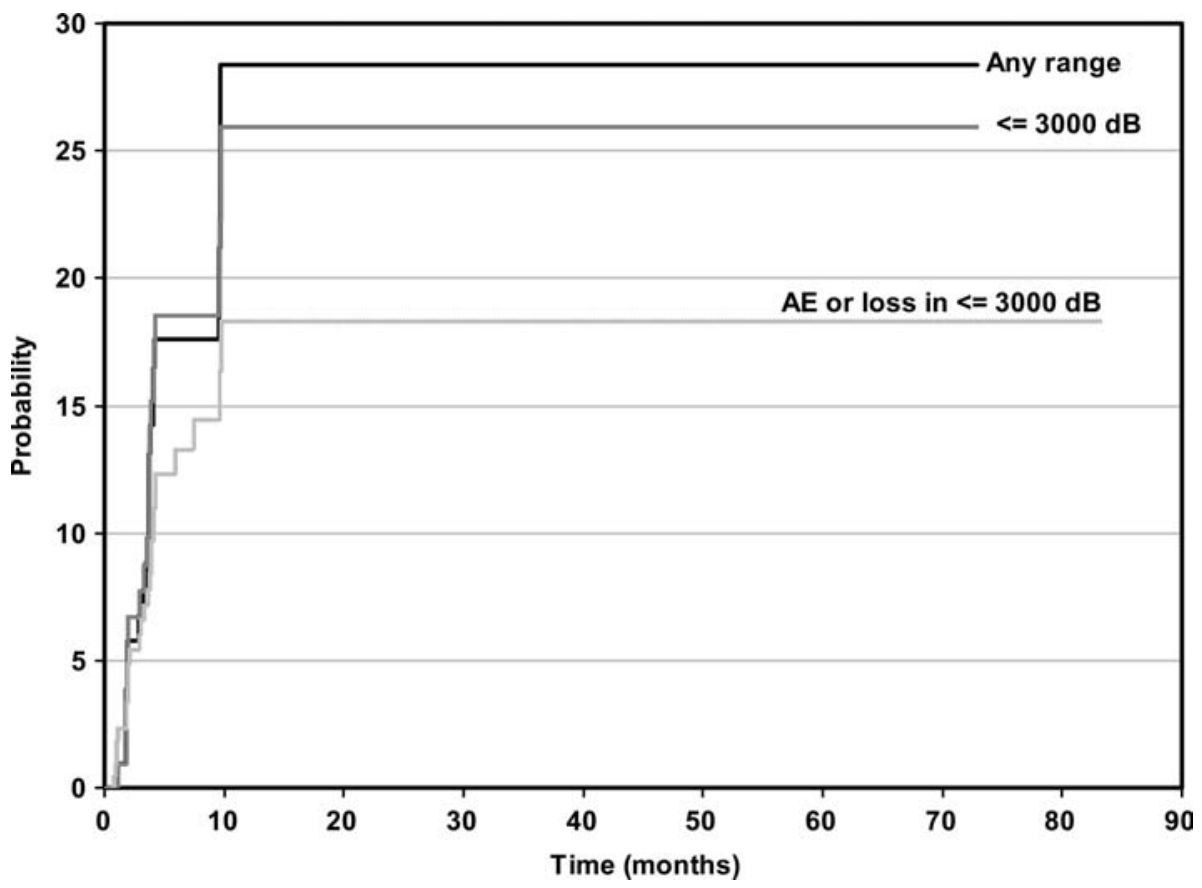

Figure 3. Audiogram findings in patients receiving radiation therapy + carmustine + cisplatin. 
because they encompass different patient groups and because the timing of the events differ for the different definitions, but rather are to be examined for changes over time. The patient group for the first two definitions are individuals who had a baseline audiogram, whereas the patient group for the last definition are all individuals on arms $\mathrm{C}$ and D. At 60 days from study enrollment, $6 \%(95 \%$ CI: $2-10)$ had a loss in at least one pure tone, $7 \%$ (95\% CI: $2-11)$ had a clinically significant loss, and 5\% (95\% CI: 2-8) had a clinically significant hearing loss and/or a hearing related adverse event of grade 3 or higher. At 6 months, these numbers increased to $18 \%(95 \% \mathrm{CI}: 10-25), 19 \%(95 \% \mathrm{CI}: 10-26)$, and $13 \%(8-18)$, respectively. Five patients were taken off-study due to ototoxicity.

Univariable Cox proportional hazards models identified age as being significantly associated with a clinically significant treatment-related hearing loss (hazards ratio: $1.06,95 \% \mathrm{CI}: 1.01-1.11, P$-value $=0.015)$. $\mathrm{No}$ other baseline variable, including the baseline audiogram frequency values, were found to be associated with a clinically significant hearing loss as determined by audiogram. Age was not found to be significantly associated with the event of a clinically significant hearing loss and/or hearing related adverse event of grade 3 or higher $(\mathrm{HR}=1.03,95 \% \mathrm{CI}: 0.99-1.07$, $P$-value $=0.11)$.

\section{Discussion}

This study found a high degree of baseline hearing loss, with $56 \%$ of patients showing at least some degree of decreased hearing. Although the majority of these hearing losses were at high frequencies, as would be expected, this prevalence of decreased hearing is greater than would be expected for patients in this age range. This raises the question of additional mechanisms of hearing loss in this patient population.

Conductive hearing loss was much less prevalent than sensorineural hearing loss. Our very limited data would seem to support that cranial radiation in GBM patients does not pose a greatly increased risk of conductive hearing toxicity. However, institutions had a much poorer compliance with reporting conductive hearing loss, which could mask significant toxicity. There were no statistically or clinically significant differences in hearing loss or ototoxicity between patients on arms $\mathrm{C}$ and $\mathrm{D}$, which suggests that accelerated radiation therapy does not increase ear damage.

Controversy remains as to the proper method to monitor cisplatin ototoxicity. Baseline screening using audiometry has frequently been recommended [18,19]. Other studies have recommended screening only patients with pre-determined risk factors, although these factors vary widely. It is as yet undetermined whether pre-treatment hearing loss does [20,21] or does not [22] contribute to additional loss. Our study found no evidence that underlying hearing loss contributed to additional loss. The only risk factor in our study shown to be statistically significant in predicting treatment damage was patient age above 58. Our data showing $56 \%$ of patients having underlying hearing loss supports the importance of baseline screening to prevent incorrect attribution of post-treatment damage. However, the relative infrequency of clinically significant hearing loss in this patient population, despite concurrent RT with CDDP, leads us to conclude that screening is most likely unnecessary and the majority of patients do not need audiograms unless they are symptomatic. Patients with clinically apparent hearing loss should receive CDDP only if there is not an appropriate alternative agent.

\section{Conclusion}

Although a large percentage of patients may show audiogram evidence of hearing loss following this treatment regimen, the percentage of patients with clinically significant ototoxicity was minimal, suggesting that screening is likely not necessary in this patient population. This study found no evidence that accelerated radiation therapy had a greater risk of ototoxicity in combination with CDDP compared to SRT, although the number of patients with follow-up audiograms precludes definitive comparisons. Increasing age is a risk factor for treatment-related hearing loss.

\section{Acknowledgements}

This study was conducted as a collaborative trial of the North Central Cancer Treatment Group and Mayo Clinic and was supported in part by Public Health Service Grant CA-25224, CA-37404, CA-15083, CA63826, CA-35103, and the National Fellowship Academic Medicine Fellowship. The authors would like to thank Drs. Christopher Bacch and Charles Beatty for their advice (Department of Otorhinolaryngology. Magoclinic, Rochester, MN).

\section{References}

1. Moroso MJ, Blair RL: A review of cis-platinum ototoxicity. J Otolaryngol 12(6): 365-369, 1983

2. Boheim K, Bichler E: Cisplatin-induced ototoxicity: audiometric findings and experimental cochlear pathology. Arch Otorhinolaryngol 242(1): 1-6, 1985

3. Schaefer SD, Post JD, Close LG, Wright CG: Ototoxicity of lowand moderate-dose cisplatin. Cancer 56(8): 1934-1939, 1985

4. Hinojosa R, Riggs LC, Strauss M, Matz GJ: Temporal bone histopathology of cisplatin ototoxicity. Am J Otolaryng 16(6): 731-740, 1995

5. Hoistad DL, Ondrey FG, Mutlu C, Schachern PA, Paparella MM, Adams GL: Histopathology of human temporal bone after cisplatinum, radiation, or both. Otolaryngol Head Neck Surg 118(6): 825-832, 1998

6. Kwong DL, Wei WI, Sham JS, Ho WK, Yuen PW, Chua DT, Au DK, Wu PM, Choy DT: Sensorineural hearing loss in patients treated for nasopharyngeal carcinoma. A prospective study of the effect of radiation and cisplatin treatment. Int J Radiat Oncol Biol Phys 36: 281-289, 1996

7. Anteunis LJ, Wanders SL, Hendriks JT, Langendijk JA, Manni JJ, de Jong JM: A Prospective Longitudinal Study on RadiationInduced Hearing Loss. Am J Surg 168: 408-411, 1994 
8. Kretschmar CS, Warren MP, Lavally BL, Dyer S, Tarbell NJ Ototoxicity of preradiation cisplatin for children with central nervous system tumors. J Clin Oncol 8: 1191-1198, 1990

9. Schell MJ, McHaney VA, Green AA, Kun LE, Hayes FA, Horowitz M, Meyer WH: Hearing loss in children and young adults receiving cisplatin with or without prior cranial irradiation. J Clin Oncol 7: 754-760, 1989

10. Stewart JD, O'Bryan RM, Al-Sarraf M, Costanzi JJ, Oishi N: Phase II study of cisplatin in recurrent astrocytomas in adults: a Southwest Oncology Group Study. J Neurooncol 1: 145-147, 1983

11. Stewart DJ, Mikhael NZ, Nair RC, Kacew S, Montpetit V, Nanji A, Maroun JA, Howard K: Platinum concentrations on human autopsy tumor samples. Am J Clin Oncol 11: 152-158, 1988

12. Douple EB: Keynote address: platinum-radiation Interactions. NCI Monograph 6: 315-319, 1988

13. Miettinen S, Laurikainen E, Johansson R, Minn H, Laurell G, Salmi TT: Radiotherapy enhanced ototoxicity of cisplatin in children. Acta Otolaryngol (Stockh) 529(Suppl): 90-94, 1997

14. Buckner JC, Michalak JC, Schomberg PJ, Burton GV, Sandler HM, Cascino TL, Hawkins RB, Scheithauer BW, O'Fallon JR Phase III trial of BCNU plus cisplatin (CDDP) versus BCNU alone, and standard radiation therapy (SRT) versus accelerated radiation therapy (ART) in glioblastoma (GMB) patients (Pts) NCCTG/SWOG results. [Abstract 219]. Proc Amer Soc Clin Oncol 20: 56a, 2001
15. Kaplan EL, Meier P: Non-parametric estimation from incomplete observations. J Am Stat Assoc 3: 457-481, 1958

16. Peto R, Peto J: Asymptotically efficient rank invariant procedures (with discussion). J Roy Stat Soc (Series A) 135: 185-207, 1972

17. Andersen PK, Gill RD: Cox's regression model for counting processes: a large sample study. Ann Stat 10: 1100-1120, 1982

18. Nagy JL, Adelsten DJ, Newman CW, Rybicki LA, Rice TW, Lavertu P: Cisplatin ototoxicity: the importance of baseline audiometry. Am J Clin Oncol 22(3): 305-308, 1999

19. Kujansuu E, Rahko T, Punnonen R, Karma P: Evaluation of the hearing loss associated with cis-platinum treatment by highfrequency audiometry. Gynecol Oncol 33(3): 321-322, 1989

20. Blakley BW, Gupta AK, Myers SF, Schwan S: Risk factors for ototoxicity due to cisplatin. Arch Otolaryngol Head Neck Surg 120: 541-546, 1994

21. Brown RL, Nuss RC, Patterson R, Irey J: Audiometric monitoring of cis-platinum ototoxicity. Gynecol Oncol 16: 254-262, 1983

22. Durrant JD, Rodgers G, Myers EN, Johnson JT: Hearing loss risk factor for cisplatin ototoxicity? Observations. Am J Otol 11: 375-377, 1990

Address for offprints: Jan C. Buckner, Mayo Clinic, 200 First Street SW, Rochester, MN, 55905, USA; Tel.: + 1-507-284-4320; Fax: +1-507-284-1803; E-mail: buckner.jan@mayo.edu 